\title{
Diagnostic and prognostic features of tuberculous meningitis on CT scanning
}

\author{
MRR B ULLOCK, * JM WELCHMAN \\ From the Department of Neurosurgery and the CT Scanning Unit, Department of Radiology, University of Natal, \\ Wentworth Hospital, Durban, South Africa
}

SUMMARY CT scans of 34 patients with severe tuberculous meningitis were reviewed. These patients were subsequently followed for a minimum period of nine months. Several diagnostic and prognostic features on CT scanning are discussed.

The diagnosis of tuberculous meningitis frequently constitutes a difficult problem. ${ }^{12}$ In view of the insidious onset of the disease many patients, particularly children, may reach referral centres as cases of coma of unknown origin. The cerebro-spinal fluid (CSF) findings of pleocytosis, raised protein and moderately depressed sugar levels constitute the most useful diagnostic pointer ${ }^{12}$ although confusion may arise because partially treated bacterial meningitis may give a similar CSF pattern, as may neurocysticercosis, in endemic areas. ${ }^{3}$ Tubercle bacilli are rarely demonstrated on Ziehl-Nielsen stains from $\mathrm{CSF}^{2}{ }^{4}$ Many patients with advanced tuberculous meningitis may have focal signs, such as a third nerve palsy, or hemiparesis, ${ }^{25}$ which may delay the performance of lumbar puncture, for fear of inducing trans-tentorial herniation due to a possible space occupying lesion. With more widespread use of CT scanning several features have been consistently described in tuberculous meningitis. ${ }^{6-13}$ We feel that many of these features may provide useful diagnostic indications (vide infra) and that CT scanning has an important role to play in tuberculous meningitis.

\section{Materials and methods}

Fifty-two patients with suspected early tuberculous meningitis were referred to this institution for CT scanning and neurological evaluation during 1980 and 1981. In 34 patients a definite diagnosis of tuberculous meningitis was made and follow-up was possible at nine months or more. The diagnosis of tuberculous meningitis was made on the basis of one or more of the following criteria: (1) demonstration of acid-fast bacilli on Ziehl-Nielsen staining of cerebrospinal fluid. (2) bromine ${ }^{82}$ partition ratio in

* Present address and address for reprint requests: Department of Neurosurgery, Institute of Neurological Sciences, Southern General Hospital, Glasgow G51 4TF, UK

Received 15 May 1982 and in revised form 25 July 1982

Accepted 31 August 19823
CSF and blood.' 14 (3) clinical and CSF responses to anti-tuberculous triple therapy. (4) culture of acid-fast bacilli from CSF (or sputum where milliary tuberculosis was present). ${ }^{2}$

CT scans performed during the first month of illness (and in some cases performed subsequently) were reviewed and correlated with the patient's initial clinical condition and response to therapy. (Isoniazid $20 \mathrm{mg} / \mathrm{kg} /$ day, ethambutol 25 $\mathrm{mg} / \mathrm{kg} /$ day, ethionamide $125-400 \mathrm{mg} /$ day, rifampicin $25 \mathrm{mg} / \mathrm{kg} /$ day, ACTH $2 \mu / \mathrm{kg} /$ day intramuscular injection).

\section{Results}

Patients were graded according to the three stages described by Lincoln et $a l^{5}$ in terms of severity of disease, as follows: Stage I: meningeal signs alone, without neurological deficit or change in conscious level, Stage II: patients with meningism and neurological deficit but with normal conscious level, Stage III: advanced cases with unconsciousness and severe neurological deficit. Only patients in stages II and III were referred for neurological evaluation and CT scanning, and were thus included in this series (table). It may be seen from fig 1 that the presence of basal lucency on CT scanning (fig $3 a$ ) carries a particularly bad prognosis in early tuberculous meningitis, being present only in patients who subsequently died or failed to improve. Periventricular lucency on CT scanning (as seen in fig 2) also carries a poor prognosis in

Table CT scan features in 34 cases of early tuberculous meningitis

\begin{tabular}{lll}
\hline Scanfeature present & $\begin{array}{l}\text { Number of } \\
\text { patients }\end{array}$ & Percentage \\
\hline Ventricular enlargement & 26 & $76 \cdot 0$ \\
Periventricular lucencies & 22 & $64 \cdot 7$ \\
Basal enhancement* & $9 / 14$ & $64 \cdot 2$ \\
Basal lucency (oedema/infarction) & 11 & $32 \cdot 3$ \\
Peripheral infarction & 7 & $20 \cdot 5$ \\
No abnormality & 8 & $23 \cdot 5$ \\
\hline
\end{tabular}

*Contrast enhancement (Conray 420) was given in a dose of $1 \mathrm{ml} / \mathrm{kg}$ bodyweight, to 14 patients only. 

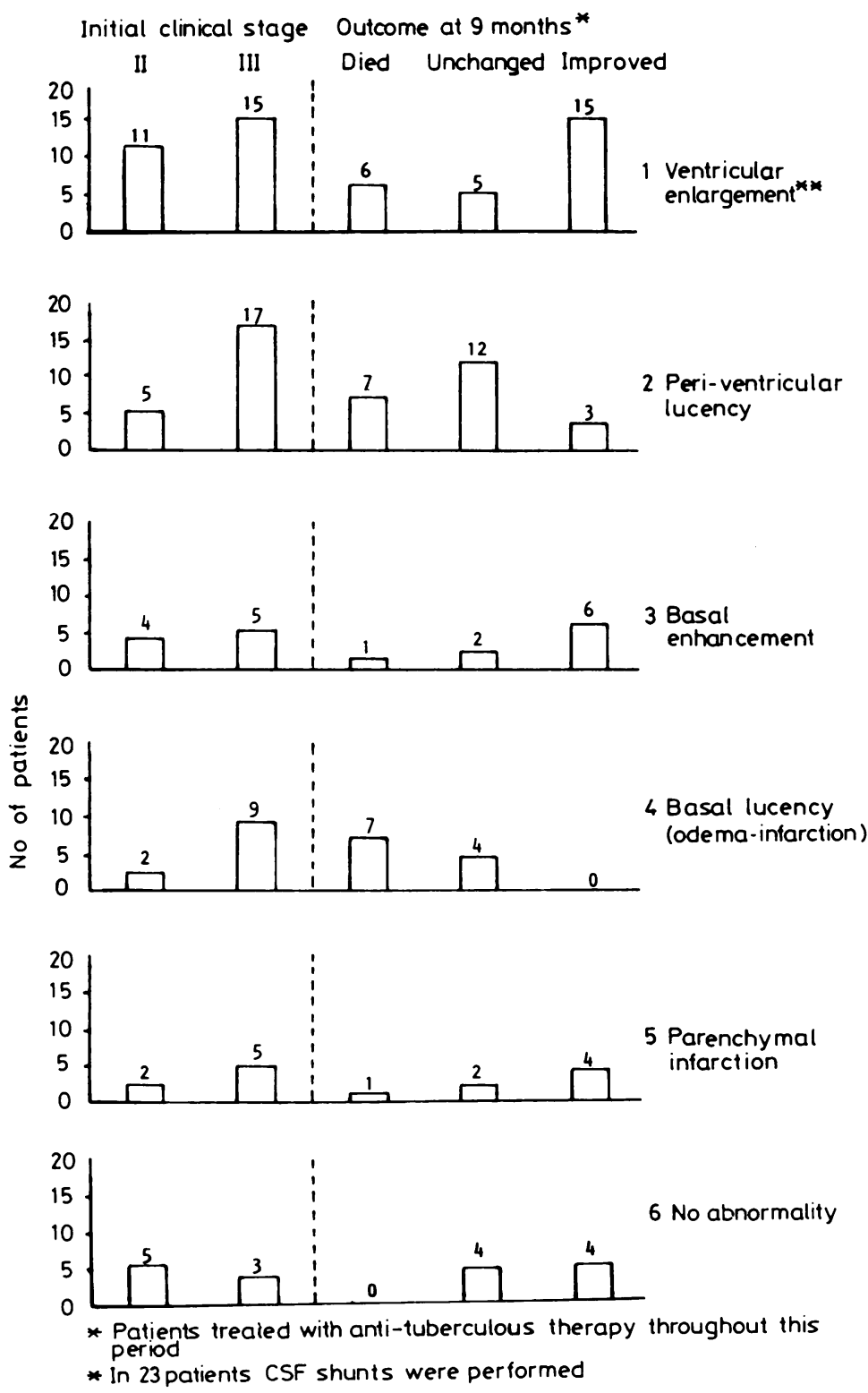

Fig I Initial clinical stage and outcome at nine months related to CT scan features. in early tuberculous meningitis.

our series. with the majority of patients remaining unchanged after nine months of chemotherapy.

The CT scan findings of ventricular enlargement alone. or basal enhancement. however (as seen in fig 2) did not constitute a bad prognostic sign. The most favourable outcome was seen in those few patients who were judged to have a normal CT scan when scanned early during the course of tuberculous meningitis.

\section{Discussion}

The most important prognostic factor for patients with tuberculous meningitis is the delay prior to initiation of anti-tuberculous chemotherapy. after onset of symptoms. 21516 We feel that CT scanning allows the diagnosis of basal meningitis to be inferred in the majority of patients who have severe early tuberculous meningitis. 


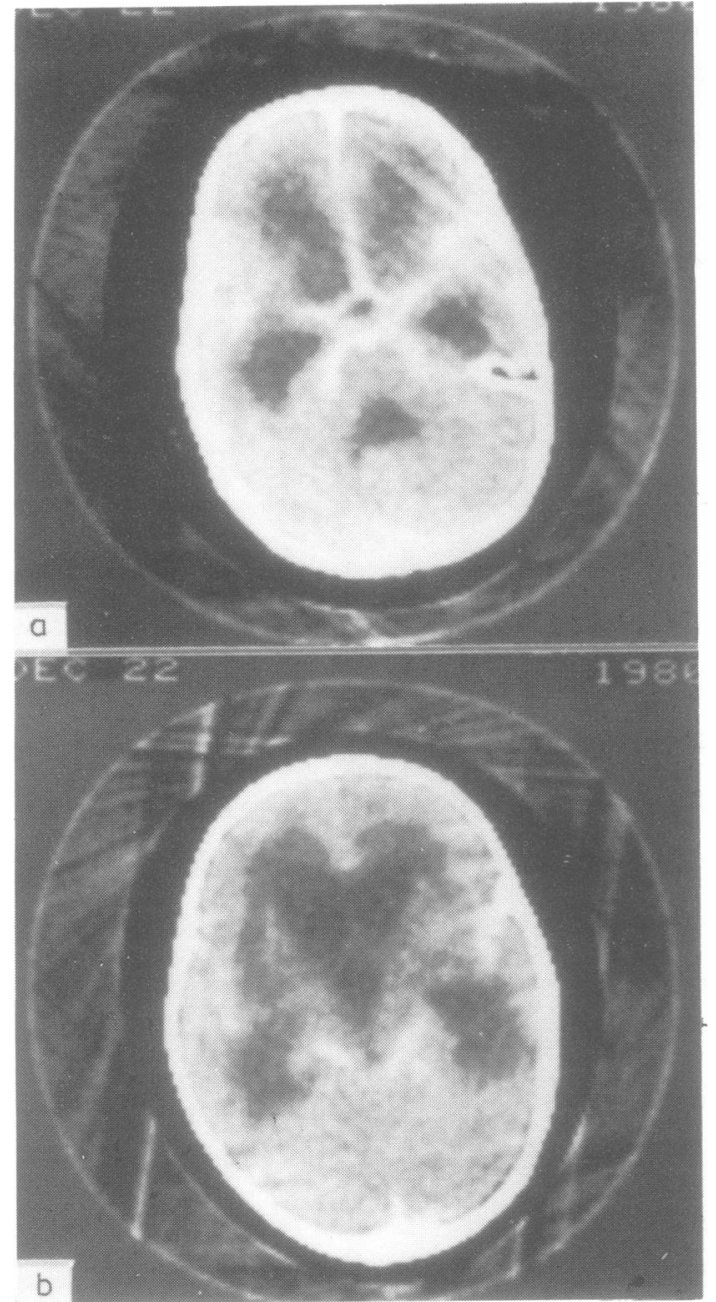

Fig 2(a,b) Enhanced CT scans demonstrating basal enhancement pattern in tuberculous meningitis, and ventricular dilation with periventricular lucencies.

and thus drug therapy may be instituted early in the disease. In addition, the demonstration of hydrocephalus and infarction provides vital therapeutic and prognostic information.

The pathological features of tuberculous meningitis are well known. ${ }^{2417} \mathrm{~A}$ characteristic thick gelatinous exudate fills the basal cisterns and envelops the basal arteries of the brain, causing a panarteritis with endarteritic infiltration. This exudate appears on CT scanning as a region of marked contrast enhancement, outlining the basal cisterns (fig 2). ${ }^{6791013}$ This CT feature has not been documented in bacterial meningitis. ${ }^{1819}$

Endarteritic vascular occlusion appears to involve predominantly the smaller perforating arteries arising
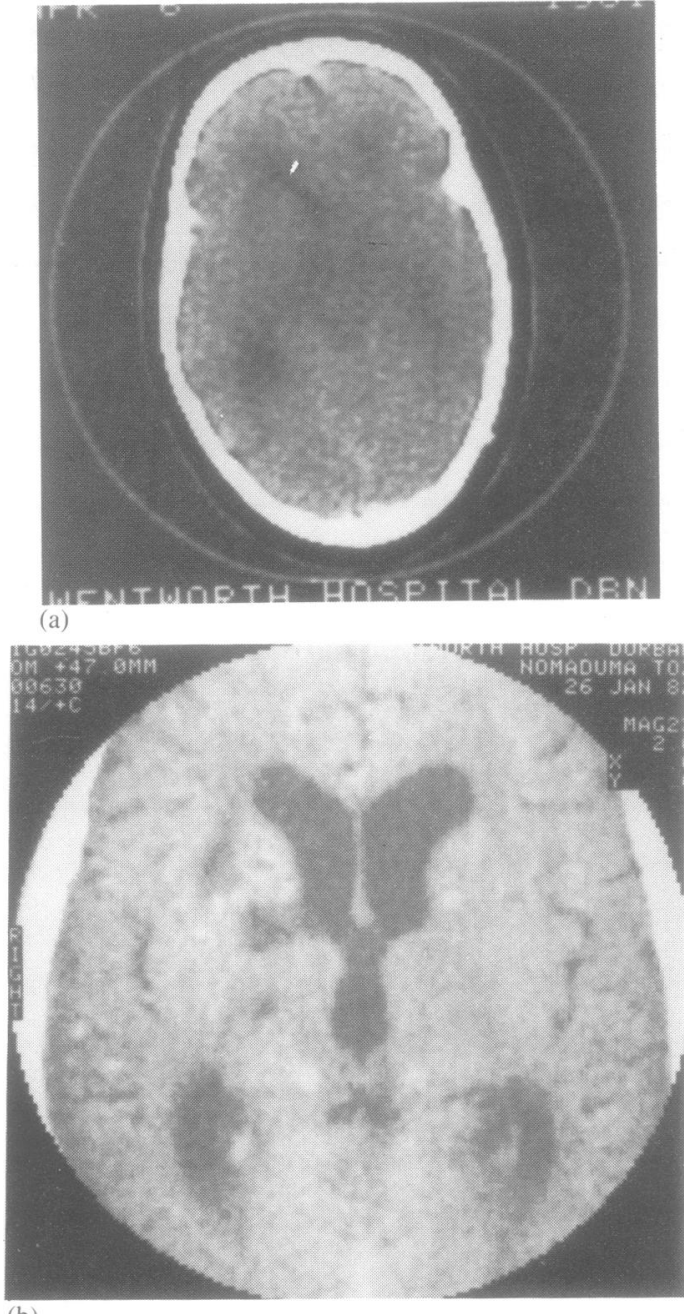

(b)

Fig 3(a) CT scan performed during early phase of severe tuberculous meningitis, demonstrating basal lucency. (b) $C T$ scan performed after three months of anti-tuberculous therapy. "Lacunar" infarcts involving the basal ganglia are demonstrated.

from the circle of Willis. In severely affected cases consequent massive diencephalic infarction causes such neurological sequelae as coma, hypertonicity and extensor rigidity, and hypothalamic dysfunction. ${ }^{41720}$ This clinical finding appears to correlate with regions of basal low density on CT scanning (fig 3). We have shown this basal "oedema" to be suggestive of a very poor prognosis in this series (fig 1). Furthermore, CT scans performed after several months in patients who have shown basal "oedema" in the early phase of tuberculous meningitis, demonstrate classical "lacunar" infarcts in the region of the basal ganglia (fig $3 b$ ). Less frequently larger vessels, 
such as middle or anterior cerebral arteries may become occluded and cause major infarction, also suggestive of a poor prognosis. ${ }^{21}$

Basal exudate causes occlusion of CSF pathways and a degree of ventricular dilatation in a high percentage of patients with advanced tuberculous meningitis ${ }^{15}(76 \%$ in our series). Many of these patients may require cerebrospinal fluid shunting. ${ }^{1522-24}$ In the majority of our patients with enlarged ventricles, periventricular lucencies were present (fig 2), the origin of which is not clear. Exudate formation and frank tubercle formation adjacent to the ventricular ependyma and choroid plexus, are frequent findings at necropsy in tuberculous meningitis, ${ }^{24}$ and periventricular lucencies on CT scanning probably reflects this direct inflammatory process. Most authors regard periventricular lucencies on CT scanning as a sign of transependymal cerebro-spinal fluid absorption, in association with raised intraventricular pressure. ${ }^{25} \mathrm{We}$ feel this may not be a reliable assumption in tuberculous meningitis, because many of our patients demonstrated low intraventricular pressure on measurement, and failed to improve with shunt procedures in the face of periventricular lucencies.

Although basal enhancement has been described in other types of basal meningitis, such as torulosis, ${ }^{1121}$ basal oedema and ventricular enlargement occurring together with basal enhancement appears to be a specific feature of advanced early tuberculous meningitis in our experience. We feel that early CT scanning, with contrast enhancement, provides a very useful diagnostic and prognostic modality in this devastating disease.

The authors acknowledge the assistance of Dr W Bulkeley of King George V Hospital Tuberculous Meningitis Unit, Durban.

\section{References}

'Mandal BK, Evans DIK, Ironside AG, Pullan BR. Radioactive bromine partition test in differential diagnosis of tuberculous meningitis. Br Med J 1972;4:413-5.

2 Tandon PN. Pathak SN. Tuberculosis of the central nervous system. In: Spillane JW, ed. Tropical neurolog: Oxford: Oxford University Press, 1973:37-51.

${ }^{3}$ Trelles JO, Trelles L. Cerebral Cysticercosis. In: Vinken PJ, Bruyn GW eds. Handbook of Clinical Neurology Volume 25. Amsterdam. Elsevier.

${ }^{4}$ Dastur DK, Lalitha VS, Udani PM, Parekh U. The brain and meninges in tuberculous meningitis. Gross pathology in 100 cases and pathogenesis. Neurology (India) 1970;18:86-92.

5 Lincoln EM, Sordillo SVR, Davies PA. Tuberculous meningitis in children. $J$ Pediat 1960;57:807-10.

${ }^{6}$ Amigo MC, Cebrian E, Castro A, Castillo J, Lema M.
Computed tomography in a verified case of tuberculous meningitis-correspondence. Neurology (Minneap) 1980;30:682.

${ }^{7}$ Arimitsu T, Jabbari B, Buckler RE. Di Chiro G: Computed tomography in a verified case of tuberculous meningitis. Neurology (Minneap) 1979;29:384-6.

${ }^{8}$ Bachman DS. Computed tomography in a verified case of tuberculous meningitis. Neurology (Minneap) 1980;30:347.

${ }^{9}$ Casselman ES, Hasso AN. Ashwal S, Schneider S. Computed tomography of tuberculous meningitis in infants and children. J Comp Assist Tomogr 1980:4(2):211-6.

${ }^{10}$ Chambers AA, Lukin RR, Thomsick TA. Cranial and intracranial tuberculosis. Semin Roentgenol 1979;14: 319-24.

${ }^{11}$ Nai-Shin CH. Tuberculous meningitis-computerized tomographic manifestations. Arch Neurol 1980;37:458-60.

12 Price HI. Danziger A. Computed tomography in cranial tuberculosis. Amer J Roentgenol 1978;130:769-71.

${ }^{1.3}$ Rovira M, Romero F. Torrent O. Ibarra B. Study of tuberculous meningitis by CT scan. Neuroradiology 1980;19:137-41.

${ }^{14} \mathrm{Da}$ Costa H. Barker A. Laken M. Distribution of orally administered Bromine ${ }^{82}$ in tubercular meningitis: concise communication. J Nucl Med 1977; 18:123-4.

15 Singhal BS, Bhagwati SN, Syed AH, Lud GW, Raised intracranial pressure in tuberculous meningitis. Neurology (India) 1975;23:32-9.

${ }^{16}$ Wilkinson HA, Ferris AJ, Muggid AL, Cantu RC. Central nervous system tuberculosis: A persistent disease. $J$ Neurosurg 1971;34:15-22.

17 Dastur DK, Udani PM. Pathology and pathogenesis of tuberculous encephalopathy. Acta Neuropath (Berl) 1966;6:311-21.

${ }^{18}$ Chaveria LE, du Boulay GH, Moseley IF. Intracranial infections: investigation by computerized axial tomography. Neuroradiology 1976;12:59-71.

${ }^{19}$ Danziger A. Price H. Schechter M. An analysis of 113 intracranial infections. Neuroradiology 1980;19:31-42.

${ }^{20}$ Kocen RS, Parsons M. Neurological complications of tuberculosis: some unusual manifestations. $Q \mathrm{~J} \mathrm{Med}$ 1970;39:17-22.

${ }^{21}$ Hendin AS, Margolis MT, Hlickman MG. Occlusion of the basilar artery in tuberculous meningitis. Neuroradiology 1973;6:193-5.

22 Bhagwati SN. Ventriculo-atrial shunt in tuberculous meningitis with hydrocephalus. J Neurosurg 1971; 35:309-13.

${ }^{2.3}$ Newman PK, Cumming WJK, Foster JB. Hydrocephalus and tuberculous meningitis in adults. $J$ Neurol Neurosurg Psychiatry 1980;43:188-90.

${ }^{24}$ Roy TK, Sircar PK, Chandar V. Peritoneal centriculo shunt in the management of tuberculous meningitis. Indian J Pediat 1979;16: 1023-7.

${ }^{25}$ Hiratsuka H, Tabata H, Tsurasoka S, Aovagi M, Okada K, Inaba $\mathrm{Y}$. Evaluation of periventricular hypodensity in experimental hydrocephalus by metrizamide CT ventriculography. J Neurosurg 1982;56:235-41. 